\title{
Oversight Authority
}

National Cancer Institute

\section{Source}

National Cancer Institute. Oversight Authority. NCI Thesaurus. Code C93445.

An org anization with monitoring, regulatory, or supervisory authority over biomedical research at the local, regional, national, or international level. 